\title{
Adiabatic state preparation in a cavity
}

\author{
J. Larson and S. Stenholm \\ Physics Department \\ Royal Institute of Technology (KTH) \\ SCFAB, Roslagstullsbacken 21 \\ SE-10691 Stockholm, Sweden
}

\begin{abstract}
The paper discusses the single-mode Jaynes-Cummings model with time dependent parameters. Solvable models for two-level systems are utilized to consider the changes in the photon distribution affected by the passage of atoms through the cavity. It is suggested that such systems may be used as filters to modify the photon distribution. The effect can be enhanced by repeatedly sending new atoms through the cavity. We show that such filters can cut out either small or large photon numbers. It is also shown that the method can be used to narrow down photon distributions and in this way achieve highly non-classical sub-Poissonian states. Some limitations and applications of the method are presented.

PACS numbers:
\end{abstract}

\section{INTRODUCTION}

Cavity QED is based on the assumption that the mode selection allows us to treat the radiation modes as a discrete set and consider states with only a few modes excited. Thus the system is described by a finite number of degrees of freedom; the rotating wave approximation (RWA) sets further limits to the states which have to be included. In the case involving a single mode only, we find the solvable Jaynes-Cummings model, which has served as a prototype for many physical situations in Quantum Optics.

The Jaynes-Cummings model was introduced in 1963 [1] and it has since played a central role in the development of Quantum Optics; see the review 2. It has also served as the theoretical foundation for analysing the results of experiments in cavity QED [3].

In all these applications, however, the model has been treated with constant parameters. In our earlier work [4], we discussed the model when the parameters are allowed to change gradually during the interaction. We now propose to continue our work on such time dependent phenomena in the framework of the Jaynes-Cummings model.

In this paper, we assume that we can consider such a case where two-level atoms consecutively interact with a single cavity mode sharp in frequency. The various decay constants are taken to be zero. Thus all time scales involved must be shorter than the damping times of the physical degrees of freedom.

In Sec. III we introduce the basic Jaynes-Cummings model with time dependent coefficients. We also discuss the possible solutions and the validity conditions for the approach. In Sec. III we illustrate the filtering action of atom dynamics by the simple results following from the Landau-Zener model. The actual discussion of the photon state filtering is given in Sec. IV where we apply the solution of the more realistic Demkov-Kunike model. We find that this may be utilised both to achieve sharpening of the photon distribution and, if we prefer, as a low or high pass photon number filter. With numerical illustrations, we show that both narrowing of the state and low pass photon filtering may be achieved. In the former case, we show that highly non-classical, sub-Poissonian photon distributions can be obtained. We also present some approximate analytical expressions for the width of the ensuing distributions. These are shown to provide god approximations to the numerically obtained exact ones. Finally we conclude the paper in Sec. $\nabla$ with a summary and some possible uses of these methods.

\section{ADIABATIC JAYNES-CUMMINGS MODEL}

In this paper, we consider the Jaynes-Cummings model with time dependent parameters. There are two essential ones; within the RWA they are the cavity detuning and the coupling constant. Both are to a large extent under experimental control in modern experiments. Here we consider the situation when these are made to change slowly during the course of the interaction between the cavity and atoms. One assumption here is, of course, that the timing of the atoms in the cavity can be controlled by the experiment. This is non-trivial but not unrealistic. The change must be slow, because we want the quantum state to follow the change adiabatically. This is necessary in order to preserve the identity of the single radiation mode involved; too fast a change will mix in higher modes and destroy the simplicity of the system. Thus we are working with an adiabatic extension of the ordinary cavity QED system.

The Jaynes-Cummings Hamiltonian separates into two-component families of coupled states, and we assume that this separation holds even when the parameters are taken to vary slowly enough. Each family is characterised by an initial photon number in the cavity, and the total solution emerges from a sum over all initial photon states. The problem now becomes one of coupled two-level systems with time dependent coefficients. Choosing these in suitable ways, we can effect a variety of transforma- 
tions on the initial photon state. We suggest this as a tool to manipulate the photon distribution and make a filter in the state space of the radiation. From the literature, however, we know that two-level systems with time dependent coefficients have played a central role in the understanding of quantum phenomena. For a review of such solvable time dependent models see the review [5]. Since a long time, many such models have been solved exactly and in this paper we are illustrating the filtering property by an application of these exact solutions.

The Hamiltonian we consider is the following one

$$
\frac{\hat{H}}{\hbar}=\Omega\left(\hat{b}^{\dagger} \hat{b}+\frac{1}{2} \sigma_{3}\right)+\frac{\Delta \omega}{2} \sigma_{3}+g\left(\hat{b}^{\dagger} \sigma^{-}+\hat{b} \sigma^{+}\right)
$$

where the Pauli matrices are defined by

$$
\begin{aligned}
& {\left[\sigma_{3}, \sigma^{ \pm}\right]= \pm 2 \sigma^{ \pm}} \\
& \sigma_{3}| \pm\rangle= \pm| \pm\rangle .
\end{aligned}
$$

The photon energy is given by $\hbar \Omega$ and the detuning is defined as

$$
\Delta \omega=\omega-\Omega
$$

where $\hbar \omega$ is the energy separation in the two-level atom. The first term in the Hamiltonian (11) is a constant of the motion, and we can thus discuss the dynamics of the other terms neglecting this first one. As usual, its effect can be added later. Here we assume that the parameters are time dependent, $g(t)$ and $\Delta \omega(t)$. The latter is presumably achieved by tuning the cavity frequency $\Omega(t)$.

We make an ansatz of the state in the form

$$
\begin{aligned}
|\Psi\rangle= & c_{0} a_{-}(0)|0,-\rangle \\
& +\sum_{n=1}^{\infty} c_{n}\left[a_{+}(n)|n-1,+\rangle+a_{-}(n)|n,-\rangle\right] .
\end{aligned}
$$

The initial states are assumed given by the coefficients $c_{n}$ and $a_{ \pm}^{0}(n)$. After the interaction period, these latter ones go over into $a_{ \pm}^{\infty}(n)$.

Without the first term of the Hamiltonian (11), we find for each family the equations of motion

$$
i \frac{d}{d t}\left[\begin{array}{c}
a_{+}(n) \\
a_{-}(n)
\end{array}\right]=\left[\begin{array}{cc}
\frac{\Delta \omega}{2} & g \sqrt{n} \\
g \sqrt{n} & -\frac{\Delta \omega}{2}
\end{array}\right]\left[\begin{array}{c}
a_{+}(n) \\
a_{-}(n)
\end{array}\right] .
$$

After a period of interaction, the solution of this problem can be written in the form

$$
\left[\begin{array}{c}
a_{+}^{\infty}(n) \\
a_{-}^{\infty}(n)
\end{array}\right]=\left[\begin{array}{cc}
\sqrt{w_{n}} & e^{-i \varphi_{n}} \sqrt{1-w_{n}} \\
-e^{i \varphi_{n}} \sqrt{1-w_{n}} & \sqrt{w_{n}}
\end{array}\right]\left[\begin{array}{c}
a_{+}^{0}(n) \\
a_{-}^{0}(n)
\end{array}\right] .
$$

If we now, after the interaction, perform a measurement to find the two-level atom in one of the states $| \pm\rangle$, we find the cavity mode to be in the corresponding state with the photon distribution

$$
\begin{aligned}
& P_{n}^{+}=\left|a_{+}^{\infty}(n+1)\right|^{2}\left|c_{n+1}\right|^{2} \\
& P_{n}^{-}=\left|a_{-}^{\infty}(n)\right|^{2}\left|c_{n}\right|^{2} .
\end{aligned}
$$

The asymmetry derives from our definition of the state (4), which allows the components in Eq.(6) to be labelled with the same $n$. With the atom emerging in the upper state, we need to start with one additional photon in the field. So the field distribution is modified by $\left|a_{ \pm}^{\infty}(n)\right|^{2}$, which we call filter functions. Before the observation of the state of the two-level atom, the normalization is given by

$$
\begin{aligned}
1=\sum_{n=0}^{\infty} P_{n}= & \sum_{n=0}^{\infty}\left[P_{n}^{+}+P_{n}^{-}\right]=\left.a_{-}(0)\right|^{2}\left|c_{0}\right|^{2} \\
& +\sum_{n=1}^{\infty}\left|c_{n}\right|^{2}\left[\left|a_{+}(n)\right|^{2}+\left|a_{-}(n)\right|^{2}\right] .
\end{aligned}
$$

After the measurement of the two-level state, the probabilities (7) must be normalized to give

$$
P^{ \pm}=\sum_{n=0}^{\infty} P_{n}^{ \pm}=1
$$

For general initial conditions, the solution (6) will depend on the phase $\varphi_{n}$ which can be quite complicated. However, the treatment simplifies if we restrict our attention to the two cases:

$$
\begin{array}{ll}
\text { Case (a): } & \left|a_{-}^{0}\right|=1 ; \quad a_{+}^{0}=0 \\
\text { Case (b) : } & a_{-}^{0}=0, \quad\left|a_{+}^{0}\right|=1 .
\end{array}
$$

In these situations, no interference terms need to be considered. In the following the initial values are taken to be as in the case (a). Since the matrix in (6) is unitairy it follows that the filter functions remain normalized, $\left|a_{-}^{\infty}(n)\right|^{2}+\left|a_{+}^{\infty}(n)\right|^{2}=1$. For the case (a), $\left|a_{-}^{\infty}(0)\right|^{2}$ does not change and hence the normalization in (8) becomes $\sum_{n=0}^{\infty}\left|c_{n}\right|^{2}=1$.

After the observation of the two-level state, we have projected out a modified photon state and applying a consecutive series of atoms with their initial states according to (10), we can iterate the transformation (7) without having to consider the phase variable. This allows us to enhance the filtering action exerted by a single atom affecting the cavity mode. It is important to point out the fact that each time an upper level atom is detected, and the photon distribution is modified by the corresponding filter function, the photon numbers are shifted by one unit as is seen in (7). 


\section{THE LANDAU-ZENER MODEL}

In the case solved by Landau and Zener, the parameters in Eq. (5) are given by

$$
\begin{aligned}
& g=g_{0}=\text { constant } \\
& \Delta \omega=2 \lambda t .
\end{aligned}
$$

The solution integrated over the time $(-\infty,+\infty)$ is given by

$$
\begin{aligned}
& w_{n}=\exp (-v n) \\
& v=\frac{\pi g_{0}^{2}}{\lambda} .
\end{aligned}
$$

For the case (a) in (10) this gives

$$
\begin{aligned}
& \left|a_{+}(n)\right|^{2}=[1-\exp (-v n)] \\
& \left|a_{-}(n)\right|^{2}=\exp (-v n) .
\end{aligned}
$$

For the case (b) the roles of the two states are interchanged. Thus for small values of the photon number, no transfer takes place, whereas for large photon numbers, the atom approaches the adiabatic limit and the state is transferred. The filter action is based on this property.

We can immediately see that the passage of a single two-level atom through the cavity effects a filtering which eliminates the low photon number states if the atom is found in its upper state and the high photon numbers if the lower state is observed. The experimentalist has, of course, no control over which is to be the case, but once the observation is done, the ensuing cavity state is determined.

In order to observe the filtering action, we assume the cavity mode initially to be in a coherent state. Then we have

$$
\left|c_{n}\right|^{2}=\exp (-\bar{n}) \frac{\bar{n}^{n}}{n !}
$$

From Eq.(7), we find that projecting on the lower state, we have the normalized distribution

$$
P_{n}^{-}=\exp \left(-\bar{n} e^{-v}\right) \frac{\left(\bar{n} e^{-v}\right)^{n}}{n !}
$$

which is a Poisson distribution with the reduced average

$$
\langle n\rangle_{-}=\exp (-v) \bar{n}
$$

Observing the other state, we find the distribution

$$
P_{n-1}^{+}=c_{N}[1-\exp (-v n)] \exp (-\bar{n}) \frac{\bar{n}^{n}}{n !}
$$

which is the difference between the original photon distribution and the one with the reduced average (16), $c_{N}$ is the normalization constant. This is clearly a method to filter out the low photon numbers. The normalization coefficient can also easily be determined from the relation

$$
P^{+}=\sum_{n=0}^{\infty} P_{n}^{+}=c_{N}\left\{1-\exp \left[\bar{n}\left(e^{-v}-1\right)\right]\right\} .
$$

After the detection of an upper level atom, the average photon number is

$$
\langle n\rangle_{+}=\frac{1-\exp \left[\bar{n}\left(e^{-v}-1\right)-v\right]}{1-\exp \left[\bar{n}\left(e^{-v}-1\right)\right]} \bar{n}-1 .
$$

If $\bar{n} \gg v$ the factor in front of $\bar{n}$ is approximately unity and $\langle n\rangle \approx \bar{n}-1$, while if $v$ is large the factor becomes greater that unity and we may have $\langle n\rangle>\bar{n}$.

If we repeatedly observe the lower state after a sequence of $m$ atoms introduced in the lower level, we obtain the result

$$
P_{n}^{-}(m)=\exp \left(-\bar{n} e^{-v m}\right) \frac{\left(\bar{n} e^{-v m}\right)^{n}}{n !},
$$

which will greatly enhance the filtering action. The corresponding distribution for the upper level is

$$
P_{n}^{+}(m) \propto\left[\prod_{\nu=1}^{m}\left|a_{+}^{\infty}(n+\nu)\right|^{2}\right] \exp (-\bar{n}) \frac{\bar{n}^{n+m}}{(n+m) !} .
$$

As each observation is totally random, the achievement of a successful series of $m$ projections will become smaller and smaller. However, the fact remains that after the observational sequence has been recorded, the state of the cavity mode is known. This holds independently of the actually observed sequence. If this contains alternating upper and lower level observations, the appropriate transformation is to be taken from (13). As the subsequent projections have to be normalized, the normalization will contain the normalization coefficients of each preceding step in the process. As long as each step involves a projection on to one of the atomic states, no phase dependence needs to be taken into account.

In order to illustrate the filtering behaviour of the Landau-Zener model, we show in figure 1, the filter function $\left|a_{-}^{\infty}(n)\right|^{2 m}$ for the repeated number of atoms, $m=1,5$ and 25 and $v=0.126$. For one atom we have the simple result, which does not provide an efficient filtering, but with increasing number of atoms, the high photon number parts become efficiently suppressed, thus pushing the average to lower and lower values. This may, for example, be used to cool an initially too hot thermal photon source. A projection on the upper level efficiently selects the high photon numbers instead.

\section{THE DEMKOV-KUNIKE MODEL}

\section{A. Results of the model}

In order to simplify the discussion, we consider only the case (a) of Eq.(10). The parameters of the model 


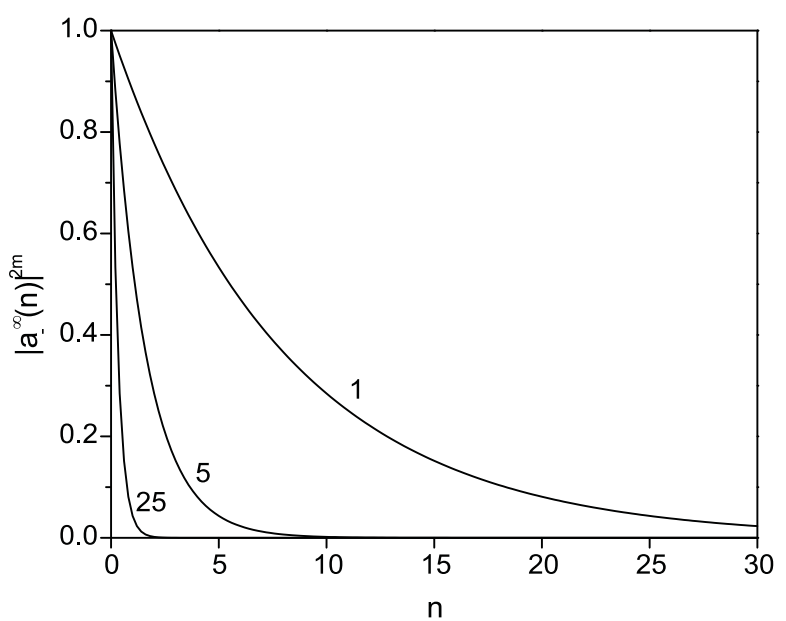

FIG. 1: This shows the filtering action achieved by the Landau-Zener model, when the outgoing atom is projected on to its lower level. The curves are presented for $v=\left(\frac{\pi g_{0}^{2}}{\lambda}\right)=$ 0.126 . The three curves give the result after the passage of one atom $(m=1)$, five atoms $(m=5)$ and twenty-five atoms $(m=25)$, which is indicated in the figure. We can easily see the increased efficiency of the filtering obtained.

given in (5) are taken as

$$
\begin{aligned}
& \Delta \omega(t)=2 A_{0} \tanh \left(\frac{t}{T}\right) \\
& g(t) \quad=g_{0} \operatorname{sech}\left(\frac{t}{T}\right) .
\end{aligned}
$$

This is a three parameter model, which integrated over the time $(-\infty,+\infty)$ gives the solution

$$
\begin{aligned}
& \left|a_{-}^{\infty}(n)\right|^{2}=\frac{\cos ^{2}\left(\pi T \sqrt{\left(g_{0}^{2} n-A_{0}^{2}\right)}\right)}{\cosh ^{2}\left(\pi T A_{0}\right)} \\
& \left|a_{+}^{\infty}(n)\right|^{2}=1-\left|a_{-}^{\infty}(n)\right|^{2} .
\end{aligned}
$$

For photon numbers such that $g_{0}^{2} n<A_{0}^{2}$, we have

$$
\left|a_{-}^{\infty}(n)\right|^{2}=\frac{\cosh ^{2}\left(\pi T \sqrt{\left(A_{0}^{2}-g_{0}^{2} n\right)}\right)}{\cosh ^{2}\left(\pi T A_{0}\right)} \rightarrow 1,
$$

as $n \rightarrow 0$. This must hold for all models as long as we consider the case (a) of Eq. (10). The filter function provided by this model, thus starts from unity, and within a range of $\left(A_{0} / g_{0}\right)^{2}$ of photon numbers goes to an oscillating function with the amplitude $\operatorname{sech}^{2}\left(\pi T A_{0}\right)$. For small coupling constants $g_{0}$, this is thus essentially a low photon number filter like the Landau-Zener case.

For a strong coupling case, $g_{0}>A_{0}$, the function is essentially an oscillating one, with the first maximum of $\left|a_{-}^{\infty}(n)\right|^{2}$ at

$$
n_{M}=\frac{\left(1+T^{2} A_{0}^{2}\right)}{T^{2} g_{0}^{2}} \sim \frac{1}{T^{2} g_{0}^{2}} .
$$

Even if the distance to the surrounding zeros is of the same order of magnitude, the trigonometric dependence on $n$ provides a rather sharp cut-off around the maximum. Application of the transformation (24) to a coherent state with most probable photon number around $n_{M}$ will result in a considerable narrowing of the photon distribution. It is advantageous to choose the parameter $A_{0}$ as small as possible, because then the oscillation amplitude $\operatorname{sech}^{2}\left(\pi T A_{0}\right)$ is maximized. The same happens when $T$ is chosen short, however, then the oscillating period becomes large. We thus want to work close to the non-adiabatic limit, of the two-level model, in this case.

In the non-adiabatic limit, the projection on the lower state of the atom gives the filtering

$$
\begin{aligned}
\left|a_{-}^{\infty}(n)\right|^{2} & =\cos ^{2}\left(\pi T \sqrt{\left(g_{0}^{2} n-A_{0}^{2}\right)}\right) \operatorname{sech}^{2}\left(\pi T A_{0}\right) \\
& \approx \cos ^{2}\left(\pi T \sqrt{g_{0}^{2} n}\right)
\end{aligned}
$$

which gives a simple filtering around the maxima

$$
n_{M}=\frac{k^{2}}{T^{2} g_{0}^{2}} . \quad(\mathrm{k}=0,1,2 \ldots)
$$

The zeros of the distribution are given by the expression

$$
n_{0}=\frac{\left(k+\frac{1}{2}\right)^{2}}{T^{2} g_{0}^{2}}
$$

The width of the distribution is hence approximated by

$$
\Delta n \sim n_{0}-n_{M} \sim \frac{k}{T^{2} g_{0}^{2}} .
$$

For a Poisson distribution, $\Delta n \sim \sqrt{\bar{n}}$. Thus to set the maximum $n_{M}$ to a large value requires a large $k$. The width (29) then scales as the square root of $\bar{n}$ with the implication that it matters little, which peak is chosen for the filtering. The factor $T g_{0}$ can well be chosen close to unity in this parameter range. One may think that for optimal filtering action, the first maximum should be chosen. However, this does not exclude large values of $n_{M}$. By a suitable choice of $T$, we can make both $A_{0} T$ and $T g_{0}$ small even if $g_{0}>A_{0}$. Thus the three parameters may be utilised to achieve desired filtering operation.

By sending atoms repeatedly through the cavity, in the case of a sequence of $m$ recorded lower level observations, we obtain the filtering

$$
P_{n}^{-}(m)=\cos ^{2 m}\left(\pi T \sqrt{g_{0}^{2} n}\right)\left|c_{n}\right|^{2} .
$$

The powers of the trigonometric function becomes rapidly sharper with increasing $m$. Thus the filtering is fast and efficient. As the repeated application of the filtering process sharpens up the photon distribution, we expect the states to rapidly become non-classical. 
In order to explore this, we have investigated the Qparameter, see [6],

$$
Q=\frac{\left\langle n^{2}\right\rangle-\langle n\rangle^{2}-\langle n\rangle}{\langle n\rangle},
$$

as a function of the coupling strength. For a range of initial coherent states, we find indeed negative values; see the next section.

In the adiabatic limit, $T$ growing large (i.e. $A_{0} T$ being large), the oscillational amplitude becomes small, and we expect the filtering process to become less efficient

$$
\operatorname{sech}^{2}\left(\pi T A_{0}\right) \sim 4 \exp \left(-2 \pi T A_{0}\right) \ll 1 .
$$

Then $\left|a_{-}^{\infty}(n)\right|^{2}$ from (23) decreases. In this case, we may also choose the weak coupling situation $g_{0}<A_{0}$ and write

$$
\begin{aligned}
P_{n}^{-}(m) & =\left(\frac{\cosh ^{2}\left(\pi T \sqrt{\left(A_{0}^{2}-g_{0}^{2} n\right)}\right)}{\cosh ^{2}\left(\pi A_{0} T\right)}\right)^{m}\left|c_{n}\right|^{2} \\
& \approx\left\{\exp \left[2 \pi T\left(\sqrt{\left(A_{0}^{2}-g_{0}^{2} n\right)}-A_{0}\right)\right]\right\}^{m}\left|c_{n}\right|^{2} \\
& \approx \exp \left(-\pi \frac{g_{0}^{2} T m}{A_{0}} n\right)\left|c_{n}\right|^{2} .
\end{aligned}
$$

This form simplifies some of the arguments below.

\section{B. Numerical results}

In this section we illustrate our argument by some numerical examples. As all relevant parameters has the dimension of time or frequency, fixing one time scale allows us to use dimensionless units in the calculations.

As we have seen, if we repeatedly project on the lower or the upper atomic level, the new photon distribution will be modified by $\left|a_{+}^{\infty}(n)\right|^{2 m}$ or $\prod_{\nu=1}^{m}\left|a_{+}^{\infty}(n+\nu)\right|^{2}$ respectively. In the latter case the initial photon distribution will also be shifted by $m$ units. In figure 2 (a) and (b) we plot the two functions above for $m=1,5$ and 25 . The maxima in (b) are shifted to the left by $m / 2$ units. For small $A_{0} T$ the function $\left|a_{-}^{\infty}(n)\right|^{2 m}$ will be approximately zero everywhere except where $n \approx n_{M}$ and $n_{M}$ is close to the maxima (27). If the initial photon distribution, given by $\left|c_{n}\right|^{2}$, has a maximum near one $n_{M}$ and a width smaller than the period of the filter function, then we expect a sharp final distribution if a series of $m$ lower level atoms is detected. We know that the $Q$-parameter defined in (31) is positive for a super-Poissonian and negative for sub-Possonian state, which signals non-classical light. A plot of $Q$ as a function of $g_{0}$ is shown in figure 3 for an initial coherent state with $\bar{n}=100$, the number of atoms $m=25$ and $T=A_{0}=0.1$. We see that the field distribution $P_{n}^{-}$is sub-Poissonian for a large range of $g_{0}:$ s. Note that one could use the $Q$-parameter to find the optimal parameters $T$ and $g_{0}$ in order to obtain the

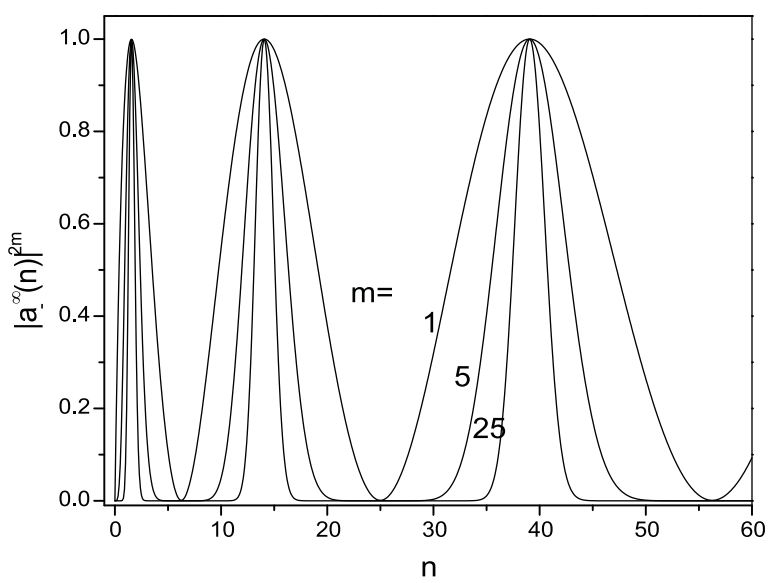

FIG. 2: This figure shows the filter functions for the DemkovKunike model. In our dimensionless units we have the parameters, $A_{0}=T=0.1$ and $g_{0}=4$. Here we also look at one, five and twenty-five atoms passing the cavity $(m=1,5,25)$. In (a) the lower level filter function $\left|a_{-}^{\infty}(n)\right|^{2 m}$ is plotted. The increase of atom number rapidly narrows the distribution, and we can also see how the width scales approximately as the position of the peak of the function. This allows us to use the filter on a coherent state distribution situated at any peak of our choice. The filter function $\prod_{\nu=1}^{m}\left|a_{+}^{\infty}(n+\nu)\right|^{2}$ is shown in (b). The maxima in (b) are shifted to the left by approximately $m / 2$ units. The numbers in the figures are again indicating how many atoms $m$ have been detected.

most non-classical light. As noted in the previous section, since the width of the maxima of the filter function goes as $\sqrt{n_{M}}$, just like the width of a coherent state, the ratio between them is independent of $n$. So if we know $g_{0}, T$ and $A_{0}$, the filtering effect is chiefly independent of which maximum $n_{M}$ the initial coherent state is centred at. The distribution $P_{n}^{-}$for an initially coherent state, centred around the third maximum $\bar{n}=25$ of the filter function, is shown in figure 4 with $T=A_{0}=0.1, g_{0}=4$ and the number of atoms $m=1,5$ and 25 .

In order to have a low photon number filter it was found to be advantageous to use a large adiabaticity parameter $\Lambda=A_{0} T$ and a small coupling constant $g_{0}<A_{0}$. A large adiabaticity parameter means that the oscillating part of the filter function is small and the weak coupling implies that the hyperbolic behaviour in the filter function extends over a long distance. In figure 5 the filter function $\left|a_{-}^{\infty}(n)\right|^{2}$ is plotted for the parameters $g_{0}=0.2$, $T=0.9$ and $A_{0}=0.6$. Except for the oscillations, this should be compared with the case $m=1$ in figure 1 .

\section{Analytical approximations}

We have considered two limits of the two-level model: The non-adiabatic one which could give a sharpened photon distribution and the adiabatic one which is used to filter out the high or the low photon number states. In 


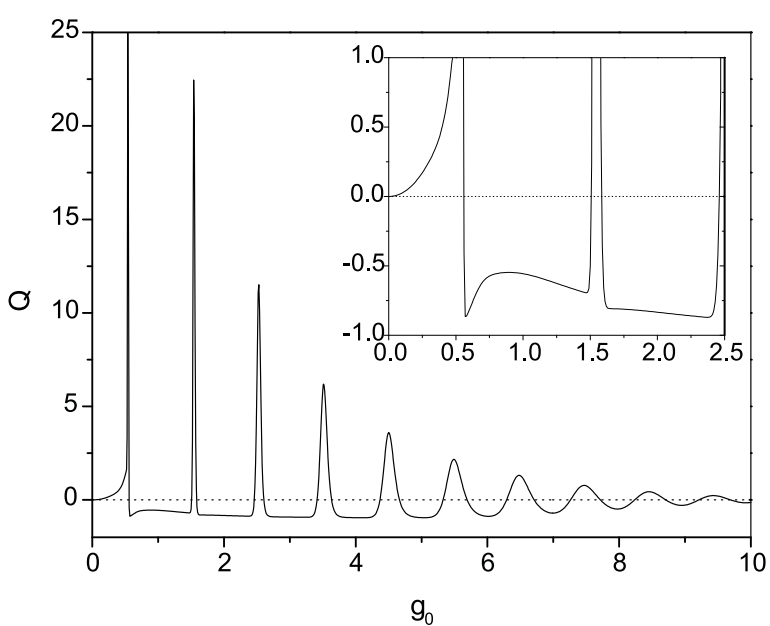

FIG. 3: This shows the variation of the Q-parameter with coupling strength. We must have $Q \geq-1$, but all negative values indicate non-classical photon statistics. The intial state assumed here has average photon number $\bar{n}=100$; the interaction is defined by $T=A_{0}=0.1$. In this figure, we consider the case with $m=25$ atoms having successfully passed the cavity and been found in their lower level. The insert zooms in on the first non-classical regions, and shows the degree of non-classicality which is achieved. The limit for classical behaviour, $Q=0$, is indicated by the dotted line.

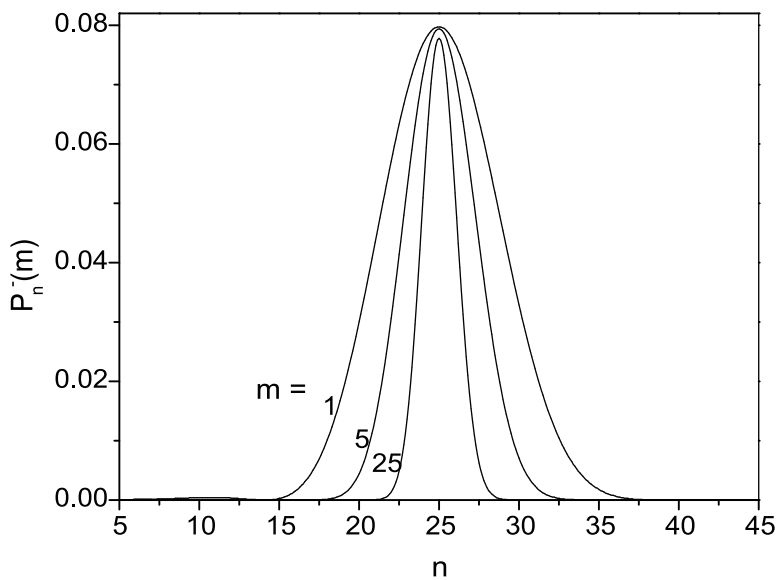

FIG. 4: This figure shows the narrowing of the phton distribution actually achieved with one, five or twenty-five atoms ( $m=1,5,25)$ acting on a coherent state centered on $\bar{n}=25$, which in this case is the second maximum; c.f. figure 2 (a). The dimensionless parameters are $T=A_{0}=0.1$ and $g_{0}=4$.

this section we derive analytic expressions for the widths of the sharpened distribution and the low photon number filter.

The filter function is given by Eq. (26) in the nonadiabatic limit, and its maximum by the result (27). After the passage of $m$ atoms, all of them detected in their lower level, we consider the photon distribution. The

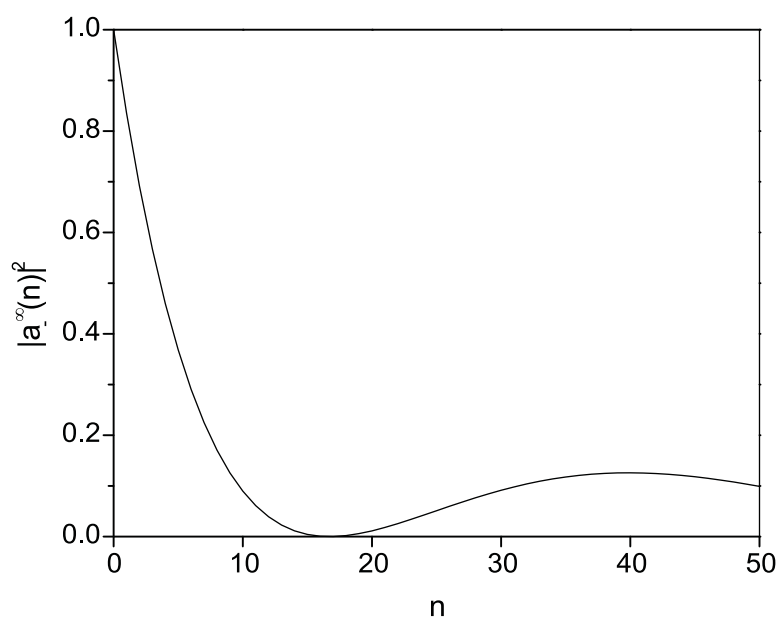

FIG. 5: This is the filtering function after the passage of one atom in the limit when the Demkov-Kunike model acts as a low photon number filter. Here $T=0.9, A_{0}=0.6$ and $g_{0}=0.2$. Except for the oscillations, this is similar to the Landau-Zener result, figure $1(m=1)$.

FWHM, $\Delta n_{a}$, of the filtering function is determined from

$$
\begin{gathered}
\cos ^{2 m}\left(\pi T g_{0} \sqrt{n_{M} \pm \Delta n_{a} / 2}\right)= \\
\cos ^{2 m}\left(n_{M} \pm \arccos \left(0.5^{(1 / 2 m)}\right)\right),
\end{gathered}
$$

where the signs \pm derive from the fact that the filtering function is not symmetric around $n_{M}$ due to the $\sqrt{n}$ behavior. Equation (34) determines two different $\Delta n_{a}$ and to get the appropiate value we take their average

$$
\Delta n_{a}=\frac{4 x_{m} \sqrt{n_{M}}}{\pi T g_{0}}
$$

where $x_{m}=\arccos (\sqrt[2 m]{0.5})$ which starts at $\pi / 4$ and falls to zero for increasing $m$. For an initial Poission distribution, we have for the half-width to a good approximation $\Delta n_{p}=\sqrt{8 n_{M} \ln (2)}$, and the width of the final distribution $P_{n}^{-}(m)$ is approximately

$$
\Delta n^{2}=\frac{\Delta n_{a}^{2} \Delta n_{p}^{2}}{\Delta n_{a}^{2}+\Delta n_{p}^{2}}=\frac{16 x_{m}^{2} n_{M} \ln (2)}{\pi^{2} T^{2} g_{0}^{2} \ln (2)+2 x_{m}^{2}} .
$$

For a narrow $\Delta n_{a}$ we have $\Delta n \approx \Delta n_{a} \sim x_{m}$. A plot of $\Delta n$ for $g_{0}=4.5, A_{0}=T=0.1$ and $\bar{n}=19.8$ as a function of $m$ is shown in figure 6 together with exact numerical results for the FWHM.

Now we turn to the adiabatic limit and the low photon number filter. With the right parameters it is possible to achieve such filtering by repeatedly projecting on the lower atomic level. The width, $\Delta n$ at $e^{-1}$, of this low photon number filter is derived from

$$
\cosh ^{2 m}\left(\pi T \sqrt{A_{0}^{2}-g_{0}^{2} \Delta n}\right) \operatorname{sech}^{2 m}\left(\pi T A_{0}\right)=e^{-1}
$$




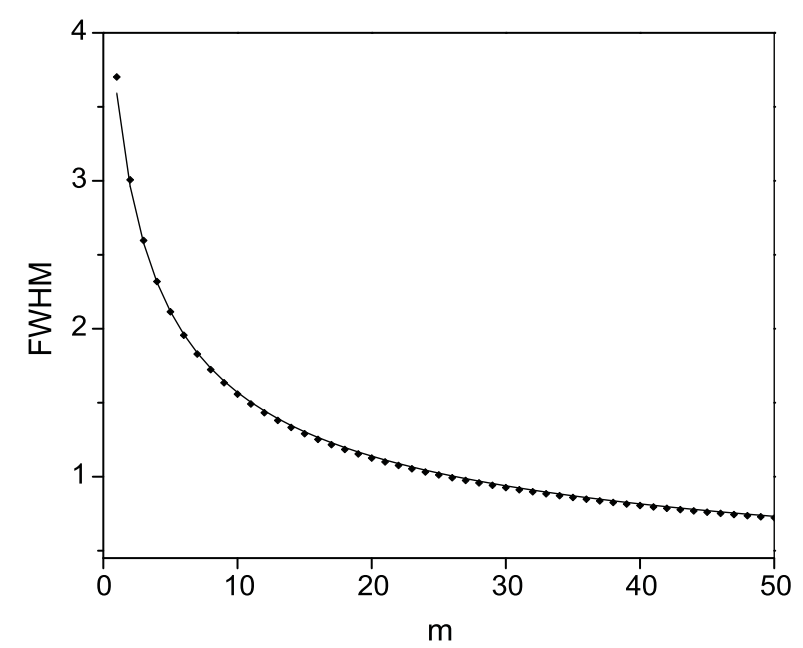

FIG. 6: This figure compares the approximate expression (36) (solid line) for the width of the final photon distribution with the exact one obtained numerically (diamonds). The dimensionless parameters are here $T=A_{0}=0.1$ and $g_{0}=4.5$. The plot shows the influence of increasing atom number $m$ on an initially coherent state with $\bar{n}=19.8$.

which determines $\Delta n$. The result is complicated and not very informative. However if we instead use the fact that we are in the adiabatic regime and take the last expression in Eq. (33) as the filter function, we obtain the width

$$
\Delta n=\frac{A_{0}}{\pi g_{0}^{2} T m}
$$

The width clearly decreases as $m$ becomes large, so that the high photon numbers are effectively filtered out. In Fig. 7 the width (38) is plotted together with the exact width obtained from Eq. (37) as a function of $m$ for the parameters $A_{0}=2$ and $T=g_{0}=1$. Again we have a very good agreement.

\section{CONCLUSION}

In this paper, we consider the situation where individual atoms are made to traverse a cavity at the will of the experimenter. During the passage of the atom through the cavity, the parameters of the model are made to vary in order to achieve a desired effect. This variation must be slow enough to preserve the energy levels of the interacting systems intact. For slow atoms this should present no problem, but the time intervals involved are limited to those allowed by the various relaxation processes which are omitted in our treatment. When actual experimental parameters are introduced, the influence of relaxation processes can easily be included by performing numerical integration of the time evolution. Here we want to present the main ideas, and such detailed investigations have been excluded.

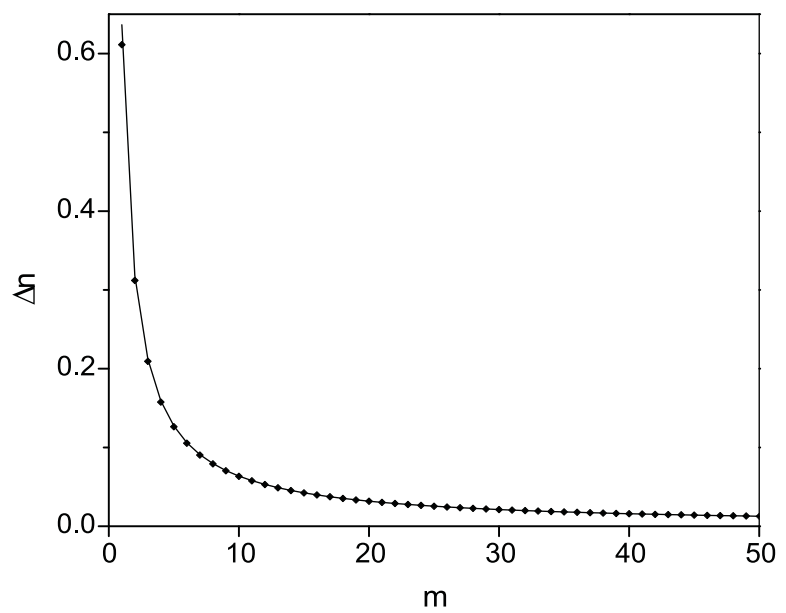

FIG. 7: This figure compares the approximate expression (38) (solid) for the width of the final photon distribution with the exact one obtained from (37) (diamonds). Here we are in the limit where the interaction acts as a low photon filter and we see the influence of increasing atom number $m$. The dimensionless parameters are $A_{0}=2$ and $T=g_{0}=1$. As we have a low photon filter, this may be interpreted as the range over which the filter acts. Alternatively we may use it as a high photon one with the corresponding interpretation.

In order to illustrate our ideas, we choose to utilise the existence of analytically solvable models for the interactions. Choosing the simple Landau-Zener model as a prototype, we then investigate in detail the behaviour for the related but much more realistic Demkov-Kunike model. They both allow filtering action for either low or high photon numbers. If low photon numbers are favoured, the process can be used to cool the photon distribution, but favouring high photon numbers we can sharpen the distribution or heat it up. For sharpening it, the DemkovKunike model provides a much more efficient tool, which we investigate in some detail. In particular, we show that strongly sub-Poissonian distributions can be achieved.

There are many treatments of time-dependent twolevel systems in the literature. Many cases have been solved analytically and the standard method is to reduce the problem to a differential equation with a known solution [7]. Such models have been utilized widely to model time-dependent atomic processes [8]. Any one of these could be used in our present scheme to analyze the result of the atom-cavity interaction. However, as we are here interested in the modification of the photon distribution, these earlier works are only of interest so far as they provide novel filter functions for the quantum field. Consequently we have here choosen to investigate two of the best known models only; they provide enough insight into the possibilties and limitations of our suggested approach. More intricate technical details of the actual solution are of little interest in the present context.

In order to obtain the most dramatic effects, we have to let several atoms pass through the cavity. Their state is 
determined experimentally after the interaction period, and the corresponding modification of the photon distribution becomes known. This occurs for either of the two possible outcomes of the experiment. However, if we want to achieve a pre-assigned series of observations, this can only be found with a certain probability. Several attempts may be necessary to observe the desired sequence. However, once it has been found, the cavity state is also known, and it may be used in any subsequent experiment within the period allowed by the cavity relaxation rates.

Our suggested approach is, of course, part of the broad range of works called "state preparation" and "state engineering". Much work has been done in this area, and we cannot possibly relate our effort to all of these. For an overview consult e.g. the special issue [9] on Quantum State Preparation and Measurement. Here we only want to point out, that the modification of the state is determined by the dynamic interaction between the atoms and the cavity mode. The repeated observation leading to projection onto the new cavity state does not, in itself, shape the distribution, it only picks one out of two possibilities.

There are many cavity QED schemes where the field in the cavity is modified by transit of atoms through the cavity, see e.g. [10] and [1] and refereces therein. Methods for prepering various states of the cavity field have been proposed, e.g. Fock states 12], Schrödinger cat states 10 and EPR states between two cavity modes [13. In these works, the time dependent feature is the transit of the atom through the cavity, and a suitable measurement at the end of the interaction probes the result achieved. The idea is, of course, closely related to our approach, but we have added the novel feature that the parameters of the coupled atom-cavity system are deliberately changed so that we achieve a desired effect. Thus the modification of the quantum state is effected by the control imposed by the experimenter and not only by the interaction followed by a detection procedeure.

We have utilized solvable models to illustrate out basic ideas. These make it possible to assert the influence of the various parameters in a straightforward way. In an actual experimental situation, we may, of course, perform the corresponding dynamical calculations on a computer, which allows one to introduce various effects related to experimental conditions and non-ideal observational methods. Such considerations are, however, best performed in connection with realistic laboratory situations.
[1] E. T. Jaynes and F. W. Cummings, Proc. IEEE 51, 89 (1963).

[2] B. W. Shore and P. L. Knight J. Mod. Opt. 40, 1195 (1993).

[3] G. Raithel, Ch. Wagner, H. Walther, L. M. Narducci and M. O. Scully, 1994, Cavity Quantum Electrodynamics, Advances in Atomic, molecular and Optical Physics, Supplement 2, , editor P. R. Berman (Academic Press), pp 57-122.

[4] F. Mattinson, M. Kira and S. Stenholm, J. Mod. Opt. 48, 889 (2001).

[5] B. M. Garraway and K.-A. Suominen, Rep. Progr. Phys. 58, 365 (1995).

[6] L. Mandel and E. Wolf, 1995, Optical Coherence and Quantum Optics, (Cambridge University Press), p 627.

[7] B. W. Shore, The Theory of Coherent Atomic Excitation, (John Wiley \& sons, New York 1990), sections 5.5 and 5.6 .

[8] E. E. Nikitin and S. Ya. Umenskii, Theory of Slow Atomic Collisions, (Springer Verlag, Heidelberg 1999), sections
7-9.

[9] W. P. Schleich and M. G. Raymer, editors, J. Mod. Opt. 44, 2021 (1997).

[10] J. M. Raimond and S. Haroche, Atoms and Cavities: The birth of a Schrödinger cat of the radiation field, in International Trends in Optics and Photonics, ed. T. Asakura (Springer Verlag, Heidelberg 1999), pp 40-53.

[11] S. Brattke, B. T. H. Varcoe and H. Walther, Phys. Rev. Lett. 86, 3534 (2001).

[12] M. Franca Santos, E. Solano and R. L. de Matos Filho, Phys. Rev. Lett. 87, 093601 (2001); S. Brattke, B. T. H. Varcoe and S. Walther, Opt. Express 8, 131 (2001); P. Bertet, S. Osnaghi, P. Milman, A. Auffeves, P. Maioli, M. Brune, J. M. Raimond and S. Haroche, Phys. Rev. Lett. 88, 143601 (2002).

[13] A. Rauschenbeutel, P. Bertet, S. Osnaghi, G. Nogues, M. Brune, J. M. Raimond and S. Haroche, Phys. Rev. A 64 050301 (2001). 